\title{
Technology Development for the Advanced Technology Large Aperture Space Telescope (ATLAST) as a Candidate Large UV- Optical-Infrared (LUVOIR) Surveyor
}

\author{
Matthew R. Bolcar*a, Kunjithapatham Balasubramanian ${ }^{\mathrm{b}}$, Mark Clampin ${ }^{\mathrm{a}}$, Julie Crooke ${ }^{\mathrm{a}}$, Lee \\ Feinberg ${ }^{\mathrm{a}}$, Marc Postman ${ }^{\mathrm{c}}$, Manuel Quijada ${ }^{\mathrm{a}}$, Bernard Rauscher ${ }^{\mathrm{a}}$, David Redding ${ }^{\mathrm{b}}$, Norman Rioux $^{\mathrm{a}}$, \\ Stuart Shaklan $^{\mathrm{b}}$, H. Philip Stahl ${ }^{\mathrm{d}}$, Carl Stahle ${ }^{\mathrm{a}}$, Harley Thronson ${ }^{\mathrm{a}}$ \\ aNASA Goddard Space Flight Center, 8800 Greenbelt Rd., Greenbelt, MD 20771; ' Jet Propulsion \\ Laboratory, 4800 Oak Grove Dr., Pasadena, CA 91109; 'Space Telescope Science Institute, 3700 \\ San Martin Dr., Baltimore, MD 21218; dNASA Marshall Space Flight Center, Redstone Arsenal, \\ Huntsville, AL 35812
}

\begin{abstract}
The Advanced Technology Large Aperture Space Telescope (ATLAST) team has identified five key technologies to enable candidate architectures for the future large-aperture ultraviolet/optical/infrared (LUVOIR) space observatory envisioned by the NASA Astrophysics 30-year roadmap, Enduring Quests, Daring Visions. The science goals of ATLAST address a broad range of astrophysical questions from early galaxy and star formation to the processes that contributed to the formation of life on Earth, combining general astrophysics with direct-imaging and spectroscopy of habitable exoplanets. The key technologies are: internal coronagraphs, starshades (or external occulters), ultra-stable large-aperture telescopes, detectors, and mirror coatings. Selected technology performance goals include: $1 \times 10^{-10}$ raw contrast at an inner working angle of 35 milli-arcseconds, wavefront error stability on the order of 10 pm RMS per wavefront control step, autonomous on-board sensing \& control, and zero-read-noise single-photon detectors spanning the exoplanet science bandpass between $400 \mathrm{~nm}$ and $1.8 \mu \mathrm{m}$. Development of these technologies will provide significant advances over current and planned observatories in terms of sensitivity, angular resolution, stability, and high-contrast imaging. The science goals of ATLAST are presented and flowed down to top-level telescope and instrument performance requirements in the context of a reference architecture: a 10-meter-class, segmented aperture telescope operating at room temperature $(\sim 290 \mathrm{~K})$ at the sun-Earth Lagrange-2 point. For each technology area, we define best estimates of required capabilities, current state-of-the-art performance, and current Technology Readiness Level (TRL) - thus identifying the current technology gap. We report on current, planned, or recommended efforts to develop each technology to TRL 5 .
\end{abstract}

Keywords: Large space telescopes, technology development, coronagraphy, direct imaging

\section{INTRODUCTION}

The 2010 Decadal Report, New Worlds, New Horizons ${ }^{l}$, recommended as its highest priority medium-scale activity a "New Worlds Technology Development Program" to "lay the technical and scientific foundations for a future space imaging and spectroscopy mission" [page 1-8]. The NASA Astrophysics 30-year roadmap, Enduring Quests, Daring Visions $^{2}$, identifies a large Ultra Violet - Optical - InfraRed surveyor (hereafter referred to as LUVOIR) as a strategic mission in the "Formative Era" (roughly the 2020's through the 2030's). The science objectives for LUVOIR include a broad array of general astrophysics pertaining to the origins of stars, planets, and galaxies, as well as the detection and characterization of habitable exoplanets. Recently, the Association of Universities for Research in Astronomy (AURA) report From Cosmic Birth to Living Earths ${ }^{3}$ recommended a 12-meter segmented-aperture space telescope, dubbed the High Definition Space Telescope (HDST), as the UV-Optical-Infrared successor to the James Webb Space Telescope (JWST). Finally, a LUVOIR surveyor concept will be studied in detail in preparation for the 2020 Decadal Survey ${ }^{4}$.

We have assembled a multi-institutional team, with members from NASA's Goddard and Marshall Space Flight Centers, the Jet Propulsion Laboratory, and the Space Telescope Science Institute, to study ATLAST, a candidate mission concept for LUVOIR and very similar in capability to the HDST. In addition to defining the science goals and

*matthew.bolcar@nasa.gov; phone 1301 286-5237; fax 1301 286-0204; www.nasa.gov/centers/goddard/home/index.html 
beginning a detailed engineering analysis, our team has produced a technology development plan that identifies the technology gaps associated with achieving the ATLAST science mission, and a set of recommended activities to begin closing those gaps in time for a new mission start in the mid-2020s. This paper presents the ATLAST technology development plan.

In Section 2 we provide an overview of the ATLAST mission concept, including top level parameters driven by the science requirements. In Sections 3-7 we present the five key technology areas, identifying the technology gaps and recommended activities for closing those gaps: Section 3-Internal Coronagraphs, Section 4-Starshades, Section 5-Ultrastable Large Aperture Telescopes, Section 6-Mirror Coatings, and Section 7-Detectors. We conclude in Section 8.

\section{THE ATLAST MISSION CONCEPT}

\section{Overview}

The science goals of ATLAST draw heavily from the documents referenced in Section 1, and are based on the scientific heritage of the Hubble Space Telescope. The investigation of the origins of cosmic structures from stars to planets to galaxies, and the mechanics of how the elemental building blocks of matter flow from galaxy to galaxy and star to star are primary science objectives. Equal in importance is the ambitious aim of detecting and spectrally characterizing dozens of habitable exoplanets and searching for biosignatures in their atmospheres. In the process of executing this search for life, ATLAST would characterize hundreds of planetary systems, enabling comparative planetology and effectively performing a census of the "exoplanet zoo." We refer the reader to AURA's From Cosmic Birth to Living Earths report for a detailed discussion of the science that can be achieved by an ATLAST-like mission ${ }^{3}$.

To help guide the engineering analysis and technology development plan, the ATLAST team is studying several reference architectures. One such architecture, shown in Figure 1, consists of a 9.2-m segmented aperture telescope that leverages JWST architecture and technology. This and other reference architectures are described in detail in a companion paper, "A Future Large Aperture UVOIR Space Observatory: Reference Designs" by Norman Rioux, et al. ${ }^{5}$
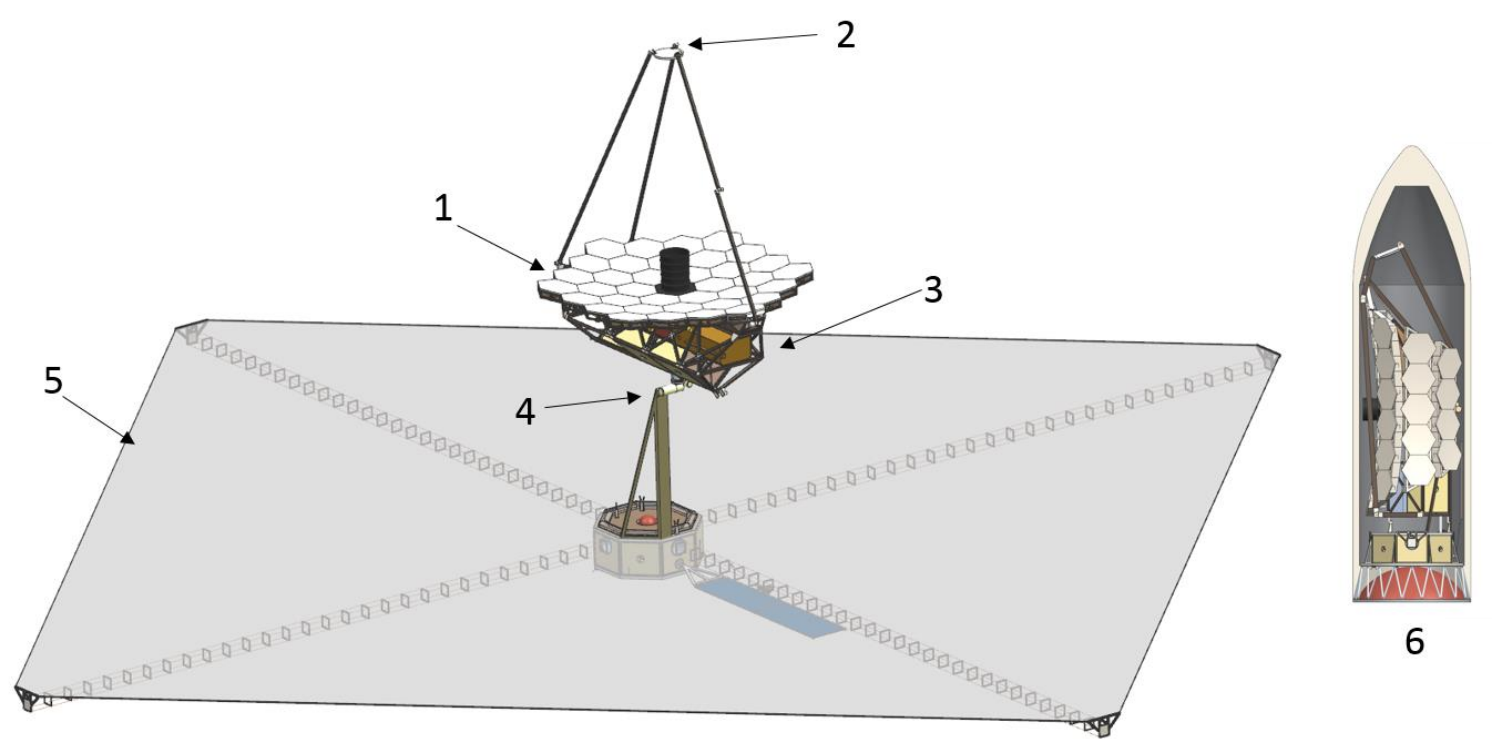

Figure 1 - The ATLAST reference architecture. The primary mirror (1) consists of 36 JWST-sized segments made from either glass or $\mathrm{SiC}$ that would use segment heater plates to maintain thermal stability. The secondary mirror (2) is actively controlled in six degrees-of-freedom using metrology referenced to the science instrument module. The science instruments (3) are externally accessible for serviceability. The entire telescope is isolated from the spacecraft via a non-contact pointing gimbal system (4). The gimbal mount allows full access to the field-of-regard while maintaining a constant viewangle between the three layer sunshield (5) and the sun, providing a stable thermal sink. Using multiple chord-folds similar to those used on JWST, the entire system can be stowed (6) inside of a typical 5-m fairing. 
Table 1 identifies the top-level telescope requirements as dictated by the science goals, and Table 2 identifies a notional list of science instruments.

Table 1 - Science requirements flow-down to the ATLAST telescope. Stretch goals are identified where mission enhancing capabilities could be realized. IR = Infrared, UV = Ultraviolet, NIR = Near-IR, MIR = Mid-IR, SNR = Signal-to-Noise Ratio, RMS = Root-Mean-Square, WFE = Wavefront Error

\begin{tabular}{|c|c|c|c|c|}
\hline \multicolumn{2}{|c|}{ Parameter } & Requirement & Stretch Goal & Traceability \\
\hline \multicolumn{2}{|c|}{ Primary Mirror Aperture } & $\geq 8$ meters & 12 meters & $\begin{array}{c}\text { Resolution, Sensitivity, } \\
\text { Exoplanet Yield }\end{array}$ \\
\hline \multicolumn{2}{|c|}{ Telescope Temperature } & $273 \mathrm{~K}-293 \mathrm{~K}$ & - & $\begin{array}{l}\text { Thermal Stability, } \\
\text { Integration \& Test, } \\
\text { Contamination, } \\
\text { IR Sensitivity }\end{array}$ \\
\hline \multirow{4}{*}{$\begin{array}{l}\text { Wavelength } \\
\text { Coverage }\end{array}$} & UV & $100 \mathrm{~nm}-300 \mathrm{~nm}$ & $90 \mathrm{~nm}-300 \mathrm{~nm}$ & - \\
\hline & Visible & $300 \mathrm{~nm}-950 \mathrm{~nm}$ & - & - \\
\hline & NIR & $950 \mathrm{~nm}-1.8 \mu \mathrm{m}$ & $950 \mathrm{~nm}-2.5 \mu \mathrm{m}$ & - \\
\hline & MIR & - & $\begin{array}{c}\text { Sensitivity to } 5.0 \mu \mathrm{m} \\
\text { under evaluation }\end{array}$ & Transit Spectroscopy \\
\hline \multirow{2}{*}{$\begin{array}{l}\text { Image } \\
\text { Quality }\end{array}$} & UV & $<0.20 \operatorname{arcsec}$ at $150 \mathrm{~nm}$ & - & - \\
\hline & Vis/NIR/MIR & Diffraction-limited at $500 \mathrm{~nm}$ & - & - \\
\hline \multicolumn{2}{|c|}{ Stray Light } & $\begin{array}{l}\text { Zodi-limited between } \\
\qquad 400 \mathrm{~nm}-1.8 \mu \mathrm{m}\end{array}$ & $\begin{array}{l}\text { Zodi-limited between } \\
200 \mathrm{~nm}-2.5 \mu \mathrm{m}\end{array}$ & $\begin{array}{l}\text { Exoplanet Imaging \& } \\
\text { Spectroscopy SNR }\end{array}$ \\
\hline \multicolumn{2}{|c|}{ Wavefront Error Stability } & $\begin{array}{l}\sim 10 \text { pm RMS uncorrected } \\
\text { system WFE per control step }\end{array}$ & - & $\begin{array}{c}\text { Starlight Suppression } \\
\text { via Internal } \\
\text { Coronagraph }\end{array}$ \\
\hline \multirow{2}{*}{ Pointing } & Spacecraft & $\leq 1$ milli-arcsec & - & - \\
\hline & Coronagraph & $<0.4$ milli-arcsec & - & - \\
\hline
\end{tabular}

A large primary mirror aperture enables high resolution and sensitivity for astrophysical observations. It has also been shown ${ }^{6}$ that the exoplanet yield is most sensitive to telescope aperture, varying as approximately $D^{1.88}$. The telescope temperature is driven by several constraints. Strict contamination requirements to enable UV observations imply the temperature should remain above freezing to avoid condensation. Furthermore, a "warm" (i.e. non-cryogenic) telescope reduces system complexity, cost, and schedule associated with mirror fabrication, integration, and testing on the ground. Conversely, the background emission of a warm telescope limits the extent into the infrared that observations can be performed.

While the two science goals of general astrophysics and exoplanet characterization are equal in importance, the highcontrast imaging that enables the exoplanet characterization is the strongest driver of wavefront stability requirements. Current internal coronagraphs require thermal and dynamic wavefront stability on the order of 10's of picometers RMS per wavefront control step.

From these top-level requirements, one can begin identifying broad technology areas that will enable mission success: a starlight suppression system capable of the required contrast at small inner working angles, a thermally and dynamically stable optical system, and high-throughput mirror coatings and sensitive detectors to enable ground-breaking UV science. Within each technology area, there are key components that contribute to the overall capability.

\section{Flexibility of Technology Development Plan}

By studying several reference architectures to help validate concepts, the ATLAST team recognizes the need to explore multiple solutions at this early stage of development. We therefore developed our technology plan to be as non-specific as possible with regards to any one architecture. For example, in the ultra-stable large-aperture telescopes technology area, we include the development of mirror technologies that are relevant to both segmented systems and large monoliths, which may prove beneficial if compatible launch vehicles become available (i.e. the Space Launch System 
(SLS) Block II configuration with an 8-10 meter fairing) and coronagraphy with segmented apertures proves too difficult.

Similarly, we also include both internal coronagraph and external occulter ("starshade") starlight suppression systems in the technology development plan. A coronagraph provides the greatest exoplanet characterization yield within a specific mission lifetime, yet it has limitations with respect to inner working angle (IWA) in the infrared. Internal coronagraphs also impose extraordinary wavefront stability requirements on the telescope. A starshade with ATLAST would still perform ground-breaking exoplanet science (albeit at reduced yields) in the event that the necessary contrast and wavefront error stability cannot be achieved with a coronagraph and a segmented aperture system. Furthermore, a follow-on starshade for the ATLAST mission could be used for extended characterization of candidate exo-Earths.

Table 2 - Science requirements flow-down to a notional instrument suite, pending engineering trades and further definition of mission science goals. The UV instrument, starlight suppression system, and exoplanet instruments would occupy the narrow on-axis field-of-view at the Cassegrain focal plane to maximize throughput. The other instruments would access the wide-field of view available at the focus of the three-mirror anastigmat system.

\begin{tabular}{|c|c|c|c|}
\hline Science Instrument & Parameter & Requirement & Stretch Goal \\
\hline \multirow{3}{*}{$\begin{array}{l}\text { UV Multi-Object } \\
\text { Spectrograph }\end{array}$} & Wavelength Range & $100 \mathrm{~nm}-300 \mathrm{~nm}$ & $90 \mathrm{~nm}-300 \mathrm{~nm}$ \\
\hline & Field-of-View & $1-2$ arcmin & - \\
\hline & Spectral Resolution & $\begin{array}{l}\mathrm{R}=20,000-300,000 \\
\text { (selectable) }\end{array}$ & - \\
\hline \multirow{3}{*}{ Visible-NIR Imager } & Wavelength Range & $300 \mathrm{~nm}-1.8 \mu \mathrm{m}$ & $300 \mathrm{~nm}-2.5 \mu \mathrm{m}$ \\
\hline & Field-of-View & $4-8$ arcmin & - \\
\hline & Image Resolution & $\begin{array}{l}\text { Nyquist sampled at } \\
500 \mathrm{~nm}\end{array}$ & - \\
\hline \multirow{3}{*}{$\begin{array}{l}\text { Visible-NIR } \\
\text { Spectrograph }\end{array}$} & Wavelength Range & $300 \mathrm{~nm}-1.8 \mu \mathrm{m}$ & $300 \mathrm{~nm}-2.5 \mu \mathrm{m}$ \\
\hline & Field-of-View & $4-8$ arcmin & - \\
\hline & Spectral Resolution & $\begin{array}{l}\mathrm{R}=100-10,000 \\
\text { (selectable) }\end{array}$ & - \\
\hline \multirow{4}{*}{$\begin{array}{l}\text { MIR Imager / } \\
\text { Spectrograph }\end{array}$} & Wavelength Range & - & $2.5 \mu \mathrm{m}-8 \mu \mathrm{m}$ \\
\hline & Field-of-View & - & $3-4$ arcmin \\
\hline & Image Resolution & - & $\begin{array}{l}\text { Nyquist sampled at } 3 \\
\mu \mathrm{m}\end{array}$ \\
\hline & Spectral Resolution & - & $\begin{array}{l}R=5-500 \\
\text { (selectable) }\end{array}$ \\
\hline \multirow{5}{*}{$\begin{array}{c}\text { Starlight Suppression } \\
\text { System }\end{array}$} & Wavelength Range & $400 \mathrm{~nm}-1.8 \mu \mathrm{m}$ & $200 \mathrm{~nm}-2.5 \mu \mathrm{m}$ \\
\hline & Raw Contrast & $1 \times 10^{-10}$ & - \\
\hline & Contrast Stability & $1 \times 10^{-11}$ over integration & - \\
\hline & Inner-working angle & 34 milli-arcsec @ 1 m & - \\
\hline & Outer-working angle & $>0.5 \operatorname{arcsec} @ 1 \mu \mathrm{m}$ & - \\
\hline \multirow{2}{*}{$\begin{array}{c}\text { Multi-Band } \\
\text { Exoplanet Imager }\end{array}$} & Field-of-View & $\sim 0.5$ arcsec & - \\
\hline & Resolution & $\begin{array}{l}\text { Nyquist sampled at } \\
500 \mathrm{~nm}\end{array}$ & - \\
\hline \multirow{2}{*}{$\begin{array}{c}\text { Exoplanet } \\
\text { Spectrograph }\end{array}$} & Field-of-View & $\sim 0.5$ arcsec & - \\
\hline & Resolution & $\mathrm{R}=70-500$ (selectable) & - \\
\hline
\end{tabular}

The extraordinary performance requirements involved in high-contrast imaging necessitate that a systems-level approach to technology development be adopted. No single technology area can be developed and evaluated independent of the others. The impact of each technology must be assessed on all of the others, supported by detailed integrated modeling 
iterations to determine what works, and what does not. Developing and validating such detailed models requires time. However, technology development must start immediately if sufficient progress is to be made in time for the 2020 Decadal Survey. We therefore adopt a conservative approach to technology development now, identifying what we believe to be the most stressing performance goals for each technology. We expect that as technologies continue to progress, and as models continue to improve our understanding of inter-relationships between technologies, some of these performance goals can be relaxed.

In the following sections, we discuss each technology area in detail, and identify the components that contribute to the overall technology area. A gap list is presented for each component that identifies the current state-of-the-art for that component, its current technology readiness level (TRL), as well as our current conservative best estimate for the performance required by ATLAST. We also classify each gap as a technology, engineering, or manufacturing gap. A technology gap indicates that a solution does not yet exist, and that a new technology must be developed to close the gap. An engineering gap indicates that existing technologies may meet the requirements with further, reasonably well understood enhancements. A manufacturing gap indicates that existing technology will meet the requirements, but fabrication processes must be developed to do so efficiently, cost effectively, and reliably.

In addition to the gap analysis, we also present recommended actions to be taken to close the gaps. These recommendations assume that each technology needs to be TRL 5 in time for a new mission start in the mid-2020's, most likely after the launch of the Wide-field Infrared Space Telescope - Astrophysics Focused Telescope Assets (WFIRST-AFTA) mission circa 2024.

\section{INTERNAL CORONAGRAPH}

\section{Overview}

The key to executing the "killer-app" science of detecting and characterizing habitable exoplanets is a starlight suppression system capable of the high-contrast imaging performance requirements, yet nimble enough to survey hundreds of planetary systems in order to achieve statistical confidence in the occurrence rate of habitable worlds. Internal coronagraphs provide this agility, but they do not yet simultaneously achieve the contrast, inner-working angle (IWA), and bandwidth requirements.

Furthermore, a >8-meter diameter aperture will almost surely be obscured, and likely segmented. Recent technology investment for the WFIRST-AFTA coronagraphs have demonstrated that current coronagraphs can be made to work in the presence of additional diffraction from aperture obscurations, but usually at the cost of throughput and Strehl ratio of the planet point-spread function (PSF). Newer, less-mature coronagraph designs show promise in meeting the demanding contrast, IWA, and bandwidth requirements while also maintaining high throughput and high planet-PSF Strehl ratio with an obscured or segmented aperture ${ }^{7,8}$.

To help mitigate some of the more stringent performance requirements of the coronagraph, it is envisioned that the instrument would operate in two modes: detection and characterization. In detection mode, the coronagraph would operate in the visible band $(\sim 550 \mathrm{~nm})$ and require higher contrast $\left(1 \times 10^{-10}\right)$, but would operate at a slightly relaxed IWA of 3.6 $\lambda / \mathrm{D}$. The detection mode would be sufficient to detect a rocky world in the habitable zone and potentially identify it as a target of interest by at least identifying coarse $\mathrm{H}_{2} \mathrm{O}$ features in the spectrum. Once a target of interest has been identified, the instrument would switch to characterization mode, in which key spectral features in the NIR are of interest. In order to operate at longer wavelengths yet still keep the planet within the high-contrast region of the focal plane, a smaller IWA of $2 \lambda / \mathrm{D}$ is required. However, the contrast ratio can be slightly relaxed to $5 \times 10^{-10}$, now that the planet has been identified and located in the focal plane. This dual-mode operation alleviates the need to have the instrument simultaneously work at the highest possible contrast and at the smallest IWA.

Similarly, we do not expect the coronagraph to simultaneously work over the entire bandwidth between $400 \mathrm{~nm}$ and 1.8 $\mu \mathrm{m}$ (or $200 \mathrm{~nm}-2.5 \mu \mathrm{m}$ bandwidth goal). Instead, we envision an instrument that is capable of work at a 10-20\% instantaneous bandwidth that can be tuned to specific central wavelengths within the broader range. This will allow for prioritization and individual characterization of key spectral features as the observation progresses.

Additional technology components contribute to the internal coronagraph's performance, including: photon-efficient wavefront sensing \& control algorithms; high-actuator count, robust deformable mirrors (DMs); autonomous highperformance onboard processing; and PSF calibration and subtraction algorithms. 


\section{Technology Gaps}

Table 3 lists the key technologies and identified gaps.

Table 3 - Internal Coronagraph technology components gap list. WFSC = wavefront sensing and control, IWA = inner working angle, OWA $=$ outer working angle, $\lambda=$ wavelength, $\mathrm{D}=$ aperture diameter, ASIC $=$ application-specific integrated circuit, GFLOPs = giga-floating-point-operations, PSF = point spread function.

\begin{tabular}{|c|c|c|c|c|c|}
\hline $\begin{array}{l}\text { Technology } \\
\text { Component }\end{array}$ & Parameter & Required & State-of-the-Art & $\begin{array}{c}\text { Current } \\
\text { TRL }\end{array}$ & $\begin{array}{l}\text { Technology, } \\
\text { Engineering, } \\
\text { Manufacturing } \\
\text { Gap }\end{array}$ \\
\hline \multirow{6}{*}{$\begin{array}{l}\text { Broadband, } \\
\text { High-Contrast } \\
\text { Coronagraph }\end{array}$} & Raw Contrast & $\begin{array}{l}1 \times 10^{-10}(\text { detect }) \\
5 \times 10^{-10}(\text { characterize })\end{array}$ & $3.2 \times 10^{-10}$ & \multirow{6}{*}{3} & \multirow{6}{*}{ Technology } \\
\hline & IWA & $\begin{array}{l}3.6 \lambda / \mathrm{D} \text { (detect) } \\
2.0 \lambda / \mathrm{D} \text { (characterize) }\end{array}$ & $3 \lambda / \mathrm{D}$ & & \\
\hline & OWA & $\sim 64 \lambda / \mathrm{D}$ & $16 \lambda / \mathrm{D}$ & & \\
\hline & Bandpass & $\begin{array}{l}10-20 \% \text { (instantaneous) } \\
400 \mathrm{~nm}-1.8 \mu \mathrm{m} \text { (total) } \\
200 \mathrm{~nm}-2.5 \mu \mathrm{m} \text { (goal) }\end{array}$ & $10 \%$ & & \\
\hline & Aperture & Obscured, Segmented & Unobscured & & \\
\hline & WFSC & $\begin{array}{l}\text { Fast WFSC at low } \\
\text { stellar photon rates to } \\
\text { generate initial dark hole } \\
\text { Low-order terms sensed } \\
\& \text { corrected to maintain } \\
1 \times 10^{-10} \text { contrast }\end{array}$ & $\begin{array}{l}\text { Multi-hour WFSC to } \\
\text { generate initial dark } \\
\text { hole, using bright } \\
\text { laboratory sources } \\
\text { Tip/tilt errors sensed } \\
\text { and corrected at sub- } \\
\text { Hertz frequencies }\end{array}$ & & \\
\hline \multirow{3}{*}{$\begin{array}{l}\text { Deformable } \\
\text { Mirrors }\end{array}$} & Actuator Count & $\begin{array}{l}128 \times 128 \text { (continuous) } \\
>3000 \text { (segmented) }\end{array}$ & $\begin{array}{l}64 \times 64 \text { (continuous) } \\
<200 \text { (segmented) }\end{array}$ & \multirow[b]{3}{*}{3} & \multirow{3}{*}{$\begin{array}{l}\text { Engineering, } \\
\text { Manufacturing }\end{array}$} \\
\hline & Environmental & Robust, radiation hard & Testing underway & & \\
\hline & Electronics & $\begin{array}{l}>16 \text { bits } \\
\text { High-speed, high- } \\
\text { throughput cabling and } \\
\text { ASICs }\end{array}$ & $\begin{array}{l}\sim 16 \text { bits } \\
\text { Dense, single-point } \\
\text { failure cables and } \\
\text { electronics }\end{array}$ & & \\
\hline \multirow{2}{*}{$\begin{array}{l}\text { Autonomous } \\
\text { Onboard } \\
\text { Processing }\end{array}$} & Bandwidth & Closed-loop $>$ a few $\mathrm{Hz}$ & $\begin{array}{l}\text { Human-in-the-loop } \\
\text { once every } 14 \text { days } \\
\text { (JWST) }\end{array}$ & \multirow{2}{*}{3} & \multirow{2}{*}{ Engineering } \\
\hline & Electronics & $\begin{array}{l}\text { Radiation hard, >100 } \\
\text { GFLOPS/W }\end{array}$ & $\begin{array}{l}<20 \text { GFLOPS/W } \\
\text { with radiation hard } \\
\text { flight electronics }\end{array}$ & & \\
\hline $\begin{array}{c}\text { Starlight } \\
\text { Suppression } \\
\text { Image } \\
\text { Processing }\end{array}$ & PSF Calibration & $\begin{array}{l}\text { Factor of } 50-100 \times \\
\text { improvement in contrast }\end{array}$ & $\begin{array}{l}25 \times \text { demonstrated } \\
30 \times \text { goal for WFIRST }\end{array}$ & 3 & Engineering \\
\hline
\end{tabular}

\section{Recommended Actions}

Internal coronagraphs are currently receiving substantial investment as part of the WFIRST-AFTA study, which should be leveraged as much as possible for ATLAST. The development of wavefront sensing algorithms and low-order wavefront sensing, robust DMs, onboard processing, and PSF calibration and subtraction can be directly applied to ATLAST's needs. However, significant additional investment is still required to bridge the gap between WFIRSTAFTA's performance requirements, and those of ATLAST. 
Notably, coronagraphs are needed that achieve an order-of-magnitude higher contrast, and they must do so while maintaining high-throughput and planet PSF Strehl in the presence of aperture obscurations and segmentation. This is perhaps the single highest-priority technology investment needed for ATLAST. As mentioned, there are several promising options here. Phase-induced amplitude apodization complex-mask coronagraphs (PIAA-CMC) ${ }^{9}$, apodized pupil Lyot coronagraphs (APLC) $)^{7}$, and phase-occulting nulling coronagraphs (PONC) ${ }^{8}$ all theoretically work with obscured and segmented apertures at high-throughput, and at least one has the potential of relaxing wavefront stability requirements on the telescope ${ }^{8}$. The PIAA-CMC concept is perhaps the most mature of these options as it has received modest investment under the WFIRST-AFTA program, but all three should be high-priorities for testbed demonstrations in the next 5 years to verify their performance.

Industry investment in higher-actuator count DMs is required to extend the OWA of the coronagraph to the outer edge of the habitable zone for nearby stars, and beyond. This will not only improve exoEarth yields, it will allow for the characterization of exoplanets that orbit beyond the habitable zone, providing a more complete "exoplanet census". A primary hurdle here is to produce large-format mirrors (e.g. $128 \times 128$ actuators), while reducing the complexity of the associated wiring harnesses and electronics. Each coronagraph is expected to use at least 2 DMs (not including additional DMs that may be part of the optical telescope assembly's aft-optics system), each with $>16,000$ wires that represent single-point failures.

Onboard processing capable of autonomously running the closed-loop wavefront sensing \& control system is required. A current desktop computer equipped with a graphics processing unit (GPUs) is more than capable of this type of computation, but it is not a flight-traceable architecture. The same performance is required in a radiation-hard system while consuming 1 to 2 orders-of-magnitude less power.

PSF calibration and subtraction algorithms will need modest investment in order to achieve a $2 \times$ improvement in performance from the WFIRST-AFTA goal.

\section{STARSHADE}

\section{Overview}

Although an internal coronagraph provides the highest exoEarth yield for an ATLAST-like mission, we recognized that it has some inherent limitations. First, despite the promise of the PIAA-CMC, APLC, and PONC concepts, it is still possible that none will achieve the required contrast, IWA, and bandwidth performance. On the other hand, it is possible that they can achieve the performance, but at the cost of unachievable wavefront stability of the telescope. In this scenario, a starshade may be the preferable starlight suppression technique.

An additional limitation for an internal coronagraph is the scaling of the IWA with $\lambda$. Assuming a 10-m aperture, a planet that is detected at $3.6 \lambda / \mathrm{D}$ at $550 \mathrm{~nm}$ moves to $\sim 2 \lambda / \mathrm{D}$ in the focal plane when observed at $1 \mu \mathrm{m}$. As the desired characterization wavelength shifts longer (where many of the interesting biomarkers exist), the coronagraph must become more aggressive and work at higher-contrast closer to the stellar-PSF core. Starshades do not have this limitation as the IWA is independent of wavelength.

We therefore include starshade development in the technology plan as both a risk mitigation in the event that an internal coronagraph proves prohibitively difficult, or as a mission enhancement to provide greater depth of exoplanet characterization in a potential future ATLAST rendezvous. The key components of this technology include the construction and demonstration of a precision large (80-m class) structure, formation flying techniques, and model validation.

We also include the need to investigate starshade servicing and propulsion options. The greatest limitation to starshades is the reduced exoEarth yields due to the finite number of slews that can be executed in a fixed lifetime - either due to fuel consumption, or to propulsion capabilities. The ability to continuously refuel a starshade provides an extendable mission lifetime (and therefore more slews). New propulsion technologies may also provide faster slews for more observations in a fixed amount of fuel. Both of these developments would provide mission-enhancing capabilities to an ATLAST-starshade concept.

\section{Technology Gaps}


Table 4 lists the key technologies and identified gaps.

Table 4 - Starshade technology components gap list.

\begin{tabular}{|c|c|c|c|c|c|}
\hline $\begin{array}{l}\text { Technology } \\
\text { Component }\end{array}$ & Parameter & Required & State-of-the-Art & $\begin{array}{c}\text { Current } \\
\text { TRL }\end{array}$ & $\begin{array}{c}\text { Technology, } \\
\text { Engineering, } \\
\text { Manufacturing } \\
\text { Gap } \\
\end{array}$ \\
\hline $\begin{array}{l}\text { Starshade } \\
\text { Construction } \\
\text { and } \\
\text { Deployment }\end{array}$ & - & $\begin{array}{l}\text { Petal and central truss } \\
\text { design consistent with } \\
\text { an } 80 \text {-m class starshade } \\
\text { Demonstrate } \\
\text { manufacturing and } \\
\text { deployment tolerances }\end{array}$ & $\begin{array}{l}\text { Demonstrated } \\
\text { prototype petal for } \\
\text { 40-m class starshade, } \\
\text { excluding blankets } \\
\text { and optical edges } \\
\text { Demonstrated } \\
\text { deployment } \\
\text { tolerances with a 12- } \\
\text { m Astromesh antenna } \\
\text { with } 4 \text { petals }\end{array}$ & 3 & Engineering \\
\hline \multirow{3}{*}{ Optical Edges } & Edge radius & $\leq 1 \mu \mathrm{m}$ & $\geq 10 \mu \mathrm{m}$ & \multirow{3}{*}{3} & \multirow{3}{*}{ Technology } \\
\hline & Reflectivity & $\leq 10 \%$ & - & & \\
\hline & Stowed radius & $\leq 1.5 \mathrm{~m}$ & - & & \\
\hline \multirow{3}{*}{$\begin{array}{l}\text { Formation } \\
\text { Flight }\end{array}$} & $\begin{array}{l}\text { Lateral sensing } \\
\text { error }\end{array}$ & $\leq 20 \mathrm{~cm}$ & - & \multirow{3}{*}{3} & \multirow{3}{*}{ Engineering } \\
\hline & $\begin{array}{l}\text { Peak-to-peak } \\
\text { control }\end{array}$ & $<1 \mathrm{~m}$ & - & & \\
\hline & $\begin{array}{l}\text { Centroid } \\
\text { estimation }\end{array}$ & $\begin{array}{l}\leq 0.3 \% \text { of optical } \\
\text { resolution }\end{array}$ & $\geq 1 \%$ & & \\
\hline $\begin{array}{c}\text { Contrast } \\
\text { Performance } \\
\text { Demonstration } \\
\text { and Model } \\
\text { Validation }\end{array}$ & - & $\begin{array}{l}1 \times 10^{-10} \text { broadband } \\
\text { contrast at Fresnel } \\
\text { numbers } \leq 50 \\
\text { Testbed performance } \\
\text { correlated with model } \\
\text { predictions. }\end{array}$ & $\begin{array}{l}3 \times 10^{-10} \text { contrast } \\
\text { excluding petal edges, } \\
\text { narrowband, at } \\
\text { Fresnel number of } \\
\sim 500 \\
\text { Model correlation is } \\
\text { good in regions } \\
\text { excluding petal edges }\end{array}$ & 3 & Technology \\
\hline $\begin{array}{l}\text { Starshade } \\
\text { Propulsion \& } \\
\text { Refueling }\end{array}$ & - & $\begin{array}{l}\text { Refueling and } \\
\text { propulsion to enable > } \\
500 \text { slews during } 3 \text { years } \\
\text { of a } 5 \text {-year mission }\end{array}$ & $\begin{array}{l}\text { Requires study; } \\
\text { Robotic refueling } \\
\text { appears feasible for } \\
\text { extended mission } \\
\text { lifetimes }\end{array}$ & 3 & $\begin{array}{l}\text { Technology, } \\
\text { Engineering }\end{array}$ \\
\hline
\end{tabular}

\section{Recommended Actions}

Once again, ATLAST is able to leverage technology development investments that have already been made through programs such as the Strategic Astrophysics Technology (SAT) program Technology Development for Exoplanets (TDEM) component. Furthermore, recent studies in a probe mission, Exo- $\mathrm{S}^{10}$, which includes a WFIRST-AFTA rendezvous concept, has spurred interest in bolstering this investment.

Similar to the internal coronagraph technology, additional investment is still required, however, to bridge the gap between a potential probe mission and what would be required for a 10-meter-class telescope such as ATLAST. The size of an ATLAST starshade would need new truss designs and deployment demonstrations. Higher-contrast would also need to be demonstrated and validated by modeling. 
As previously mentioned, propulsion and refueling technologies should be investigated to help improve exoEarth yields. Robotic refueling has been extensively studied and demonstrated in low-Earth orbit ${ }^{11}$. A study of the appropriate trade space is required, addressing such questions as: "Which services are required?" (refueling, repair, replacement, etc.); "Where should servicing be performed?" (Low-earth orbit, cis-lunar space, SEL2, etc.); "How much fuel is needed, and how often?"; and "What infrastructure exists, and what will be required?". New propulsion technologies, including solar-electric propulsion, should be investigated to enable faster slews.

\section{ULTRA-STABLE LARGE APERTURE TELESCOPE}

\section{Overview}

One might think that after starlight suppression techniques, the highest priority technology needed for a large aperture space telescope would be the mirrors that constitute the optical system. However, the extraordinary wavefront stability requirements levied on an observatory by an internal coronagraph necessitate that mirror technology development involves more than just mirrors. Instead, the optical telescope assembly (OTA) must be developed as a system, including: mirrors, structures, disturbance isolation and damping, metrology, actuators, and thermal control systems.

As noted earlier, we adopt a conservative estimate on the requirements for wavefront stability to which each of these technologies contribute. Assuming the high-contrast exoplanet science is performed with an internal coronagraph, the top-level requirement for wavefront stability is currently understood to be 10's of picometers RMS per wavefront control step $^{12}$. This is based on the current set of coronagraphs being developed for the WFIRST-AFTA mission. As mentioned in Section 3, however, we note the development of new coronagraphic techniques that may help relax this requirement. Furthermore, the control frequency of the wavefront sensing and control system is currently unknown, as it depends on the specific coronagraph architecture, observing strategy, wavefront sensing system, and brightness of the host star. As these are still open-ended trades to be performed, we desire to make the telescope as stable as possible to accommodate the possibility of a slow wavefront control system coupled with a demanding coronagraphic instrument.

\section{Technology Gaps}

Table 5 lists the key technology components and the identified gaps.

Table 5 - Ultra-stable Large Aperture Telescopes technology components gap list. DIVH = Delta IV-Heavy, SLS = Space Launch System, $\mathrm{SiC}=$ Silicon Carbide, MMSD = Multiple Mirror System Demonstration, TMT $=$ Thirty Meter Telescope, WFC $=$ Wavefront Control

\begin{tabular}{|c|c|c|c|c|c|}
\hline $\begin{array}{l}\text { Technology } \\
\text { Component }\end{array}$ & Parameter & Required & State-of-the-Art & $\begin{array}{l}\text { Current } \\
\text { TRL }\end{array}$ & $\begin{array}{c}\text { Technology, } \\
\text { Engineering, } \\
\text { Manufacturing } \\
\text { Gap }\end{array}$ \\
\hline \multirow{4}{*}{ Mirrors } & Surface Figure & $<7 \mathrm{~nm}$ RMS & $<7 \mathrm{~nm} \mathrm{RMS}$ & \multirow{4}{*}{4} & \multirow{4}{*}{$\begin{array}{l}\text { Engineering, } \\
\text { Manufacturing }\end{array}$} \\
\hline & Areal Density & $\begin{array}{l}<36 \mathrm{~kg} / \mathrm{m}^{2}(\text { DIVH }) \\
<500 \mathrm{~kg} / \mathrm{m}^{2}(\mathrm{SLS})\end{array}$ & $\begin{array}{l}\sim 12 \mathrm{~kg} / \mathrm{m}^{2}(\mathrm{SiC} \\
\mathrm{w} / \text { nanolaminates}) \\
\sim 35 \mathrm{~kg} / \mathrm{m}^{2} \text { (MMSD) } \\
70 \mathrm{~kg} / \mathrm{m} 2 \text { (JWST) }\end{array}$ & & \\
\hline & Areal Cost & $<\$ 2 \mathrm{M} / \mathrm{m}^{2}$ & $\$ 6 \mathrm{M} / \mathrm{m}^{2}(\mathrm{JWST})$ & & \\
\hline & $\begin{array}{l}\text { Areal Production } \\
\text { Rate }\end{array}$ & $30-50 \mathrm{~m}^{2} /$ year & $\begin{array}{l}\sim 4 \mathrm{~m}^{2} / \text { year (JWST) } \\
\sim 1 \mathrm{~m}^{2} / \text { year (HST) } \\
\sim 100-300 \mathrm{~m}^{2} / \text { year } \\
\text { planned by TMT but } \\
\text { not yet demonstrated }\end{array}$ & & \\
\hline \multirow{2}{*}{$\begin{array}{l}\text { Composite } \\
\text { Material } \\
\text { Structures }\end{array}$} & $\begin{array}{l}\text { Coefficient of } \\
\text { Moisture } \\
\text { Expansion }\end{array}$ & $\begin{array}{l}\text { Constant after initial } \\
\text { moisture release }\end{array}$ & $\begin{array}{l}\text { Continuous moisture } \\
\text { release }\end{array}$ & \multirow[t]{2}{*}{3} & \multirow[t]{2}{*}{ Technology } \\
\hline & Lurch & $<10 \mathrm{pm} / \mathrm{WFC}$ step & Micro-lurch at joints & & \\
\hline
\end{tabular}




\begin{tabular}{|c|l|l|l|c|l|}
\hline $\begin{array}{c}\text { Thermal } \\
\text { Stability }\end{array}$ & Material Stability & $\sim 10 \mathrm{~nm} / \mathrm{K}$ & $\sim 100 \mathrm{~nm} / \mathrm{K}$ & 3 & Technology \\
\hline $\begin{array}{c}\text { Disturbance } \\
\text { Isolation } \\
\text { System }\end{array}$ & $\begin{array}{l}\text { End-to-end } \\
\text { Attenuation }\end{array}$ & $\begin{array}{l}140 \mathrm{~dB} \text { at frequencies } \\
>20 \mathrm{~Hz}\end{array}$ & $\begin{array}{l}80 \mathrm{~dB} \text { at frequencies } \\
>40 \mathrm{~Hz} \text { (JWST } \\
\text { passive isolator only) }\end{array}$ & 4 & $\begin{array}{c}\text { Technology, } \\
\text { Engineering }\end{array}$ \\
\hline $\begin{array}{c}\text { Metrology \& } \\
\text { Actuators }\end{array}$ & Sensing Accuracy & $\sim 1 \mathrm{pm}$ & $\sim 1 \mathrm{~nm}$ & \multirow{2}{*}{4} & Technology \\
\cline { 2 - 4 } & Control Accuracy & $\sim 1 \mathrm{pm}$ & $\sim 5 \mathrm{~nm}$ & & \\
\hline
\end{tabular}

\section{Recommended Actions}

Development of these technologies requires a systems-level approach, and it must be driven by detailed integrated modeling to validate designs. However, there are a few concrete steps that can be taken to advance the TRL of the technology components.

A mirror development program similar to the Advanced Mirror System Demonstration ${ }^{13}$ (AMSD) program is recommended. Multiple mirror materials (ULE ${ }^{\circledR}$, Zerodur ${ }^{\circledR}, \mathrm{SiC}$, composite, etc.) and mirror architectures (open vs. closed back, monolith vs. rigid-body segments vs. high-authority actuated segments, etc.) should be evaluated and compared. Candidate downselects should be performed every 3-5 years until two candidates remain for contract competition. Throughout this process, production should be maintained to ensure that expertise is retained.

Subscale demonstrations of composite structures should be used to advance the development of new composite materials with lower outgassing and new joint designs to reduce micro/nano-lurch properties. Perhaps most importantly, testing a subscale structure at the picometer level will validate linearity assumptions that are commonly used in integrated modeling. Once validated, integrated modeling can then be used to accurately predict the performance of full-scale systems.

A distributed tiered-approach to thermal control needs to be studied and implemented. Elements of the thermal control system could include: (1) thermally stable mirror(s) with uniform coefficient of thermal expansion (CTE) distributions; (2) new thermally stable composite material structures; (3) observatory-level architecture trades between flat sunshields a la JWST or stray-light barrels a la HST; (4) new thermal sensing and control schemes to manage residual thermal instabilities.

The advancement of industry-developed non-contact isolation technology, which has the potential of meeting the stringent dynamic stability requirements, should also be prioritized. Demonstrating the required isolation with this type of system at TRL 6, including power and data transmission across the bus-payload interface, would retire a key risk. It is also important to investigate other potential isolation and pointing techniques (i.e. reaction wheel isolation systems, micro-thrusters, etc.) that may be combined to meet the dynamic stability requirements in order to increase margin and reduce overall system cost and risk.

Similar to the thermal control system, a tiered approach to metrology and wavefront control is needed. Components such as image-based wavefront sensing, laser metrology, or capacitive edge sensors should be studied in combination to detect dynamic disturbances over various temporal and spatial frequencies. Primary and secondary mirror control via segment-level rigid-body actuators or embedded high-authority actuators may be paired with deformable mirrors in the instrument payload to provide the picometer-level control needed for high-contrast imaging.

\section{MIRROR COATINGS}

\section{Overview}

A UVOIR telescope such as ATLAST will require protected aluminum coatings very similar to those employed on HST, which are arguably already TRL 9. However, some mission-enhancing gains in coating performance can be realized via better coating deposition processes. Furthermore, some new study is required to fully understand the impact that polarization effects may have on coronagraph performance. 
Four areas of improvement are recommended: higher reflectivity (specifically in the Far-UV); better coating uniformity across the entire UVOIR bandpass; lower polarization (specifically over the exoplanet science band between $400 \mathrm{~nm}$ and $1.8 \mu \mathrm{m})$; and better durability overall.

\section{Technology Gaps}

Table 6 lists the key technology components and identified gaps.

Table 6 - Mirror Coatings technology components gap list.

\begin{tabular}{|c|c|c|c|c|c|}
\hline $\begin{array}{l}\text { Technology } \\
\text { Component }\end{array}$ & Parameter & Required & State-of-the-Art & $\begin{array}{c}\text { Current } \\
\text { TRL }\end{array}$ & $\begin{array}{c}\text { Technology, } \\
\text { Engineering, } \\
\text { Manufacturing } \\
\text { Gap } \\
\end{array}$ \\
\hline \multirow{3}{*}{ Reflectivity } & $90-120 \mathrm{~nm}$ & $>70 \%$ & $<50 \%$ & \multirow{3}{*}{$\begin{array}{l}2 \\
3 \\
5 \\
\end{array}$} & \multirow{3}{*}{$\begin{array}{l}\text { Technology, } \\
\text { Engineering }\end{array}$} \\
\hline & $120-300 \mathrm{~nm}$ & $>90 \%$ & $80 \%$ & & \\
\hline & $>300 \mathrm{~nm}$ & $>90 \%$ & $>90 \%$ & & \\
\hline \multirow{3}{*}{ Uniformity } & $90 \mathrm{~nm}-120 \mathrm{~nm}$ & $<1 \%$ & TBD & \multirow{3}{*}{$\begin{array}{l}2 \\
2 \\
3\end{array}$} & \multirow{3}{*}{$\begin{array}{l}\text { Engineering, } \\
\text { Manufacturing }\end{array}$} \\
\hline & $120 \mathrm{~nm}-250 \mathrm{~nm}$ & $<1 \%$ & $>2 \%$ & & \\
\hline & $>250 \mathrm{~nm}$ & $<1 \%$ & $1-2 \%$ & & \\
\hline Polarization & $\geq 90 \mathrm{~nm}$ & $<1 \%$ & $\begin{array}{l}\text { Not yet assessed; } \\
\text { requires study }\end{array}$ & 2 & Technology \\
\hline Durability & - & $\begin{array}{l}\text { Stable performance } \\
\text { over mission lifetime } \\
\text { (10 years minimum) }\end{array}$ & $\begin{array}{l}\text { Stable performance, } \\
\text { but with limited } \\
\text { starting reflectivity } \\
\text { below } 200 \mathrm{~nm}\end{array}$ & 4 & $\begin{array}{l}\text { Engineering, } \\
\text { Manufacturing }\end{array}$ \\
\hline
\end{tabular}

\section{Recommended Actions}

It is unlikely that much will change in the fundamental technology of coatings - by far the best coating for a broad UVOIR bandpass is aluminum protected by a thin dielectric layer (typically $\mathrm{MgF}_{2}, \mathrm{LiF}$, or $\mathrm{AlF}_{3}$ ). Instead, investments should be focused on improving deposition processes for these materials. New techniques in atomic layer deposition (ALD) and ion-beam assisted physical vapor deposition (IBAPVD) can improve material packing densities, resulting in higher reflectivity and better uniformity. Importantly, scaleable processes that can be applied to meter-class segments and 8-meter-class monoliths should be developed.

The effects of coating polarization on high-contrast imaging with a coronagraph is an issue that needs to be addressed regardless of what coating is used. This is also an area where technology development for WFIRST-AFTA can be leveraged. Even though its coatings are protected silver, polarization is still induced in the collected light. A thorough understanding of how the coatings affect polarization and how polarization affects high-contrast imaging is needed. If the effects are significant, a mitigation plan is needed to implement polarization control/correction in the coronagraph.

\section{DETECTORS}

\section{Overview}

The habitable exoplanet detection and characterization component of ATLAST is enabled by improvements in extremely low-noise, single photon detectors in the Visible-NIR band $(400 \mathrm{~nm}-1.8 \mu \mathrm{m})$. While many robust technologies exist in this area, additional testing for radiation hardness and further reductions in noise and dark count rate are required. This science goal is further enhanced by developments of extremely low-noise, single photon detectors in the UV (200 nm $400 \mathrm{~nm}$ ). Such detectors would allow for spectral characterization of the important ozone biomarker feature between $260 \mathrm{~nm}-350 \mathrm{~nm}$.

The general astrophysics component ATLAST can be enhanced by improvements in large-format, high-sensitivity, radiation-hard UV detectors. To take advantage of the resolution afforded by a large-aperture system, higher-pixel-count detectors are needed to cover desired fields-of-view. Furthermore, current UV detector technologies are limited in sensitivity at all wavelengths, but especially below $150 \mathrm{~nm}$, where critical science goals exist. Some applications also benefit from "visible blind" detectors with little or no sensitivity above $300 \mathrm{~nm}$. 
In all cases, it is extremely desirable to avoid the need for cryogenic operation that necessitates a cryo-cooler in order to minimize cost, complexity, mass, and vibration that may impact wavefront stability for high-contrast exoplanet science.

\section{Technology Gaps}

Table 7 lists the key technology components and identified gaps.

Table 7 - Detector technology components gap list. EMCCD = electron multiplying charge coupled device, SEL2 = sunearth Lagrange 2, APD = avalanche photo-diode, MKID = microwave kinetic inductance detector, TES = transition edge sensor, EBCMOS = electron bombarded complementary metal-oxide semiconductor, MCP = micro-channel plate.

\begin{tabular}{|c|c|c|c|c|c|}
\hline $\begin{array}{l}\text { Technology } \\
\text { Component }\end{array}$ & Parameter & Required & State-of-the-Art & $\begin{array}{c}\text { Current } \\
\text { TRL }\end{array}$ & $\begin{array}{c}\text { Technology, } \\
\text { Engineering, } \\
\text { Manufacturing } \\
\text { Gap }\end{array}$ \\
\hline \multirow{7}{*}{$\begin{array}{l}\text { Vis-NIR } \\
\text { Single Photon } \\
\text { Detectors for } \\
\text { Enabling } \\
\text { Exoplanet } \\
\text { Science }\end{array}$} & $\begin{array}{l}\text { Operational } \\
\text { Bandwidth }\end{array}$ & $\begin{array}{l}400 \mathrm{~nm}-1.8 \mu \mathrm{m} \\
(2.5 \mu \mathrm{m} \text { goal })\end{array}$ & \multirow{7}{*}{$\begin{array}{l}\text { EMCCD technology } \\
\text { is promising, but } \\
\text { requires radiation } \\
\text { hardness testing, and } \\
\text { have a hard cutoff at } \\
1.1 \mu \mathrm{m} \text {; } \\
\mathrm{HgCdTe} \text { APDs are } \\
\text { candidates for NIR } \\
\text { but need better dark } \\
\text { count rates; } \\
\text { MKID and TES meet } \\
\text { requirements, but } \\
\text { need cryogenic } \\
\text { temperatures }\end{array}$} & \multirow{7}{*}{$3-5$} & \multirow{7}{*}{$\begin{array}{l}\text { Technology, } \\
\text { Engineering, } \\
\text { Manufacturing }\end{array}$} \\
\hline & Read Noise & $<<1 \mathrm{e}^{-}$ & & & \\
\hline & Dark Current & $<0.001 \mathrm{e}^{-} / \mathrm{pix} / \mathrm{s}$ & & & \\
\hline & $\begin{array}{l}\text { Spurious Count } \\
\text { Rate }\end{array}$ & $\begin{array}{l}\text { Small compared to } \\
\text { dark current }\end{array}$ & & & \\
\hline & $\begin{array}{l}\text { Quantum } \\
\text { Efficiency }\end{array}$ & $\begin{array}{l}>80 \% \text { over entire } \\
\text { band }\end{array}$ & & & \\
\hline & Format & $>2 \mathrm{k} \times 2 \mathrm{k}$ pixels & & & \\
\hline & Other & $\begin{array}{l}\text { Radiation hard, } \\
\text { minimum 5-year } \\
\text { lifetime at SEL2, } \\
\text { Non-cryogenic } \\
\text { operation preferable }\end{array}$ & & & \\
\hline \multirow{7}{*}{$\begin{array}{l}\text { UV Single } \\
\text { Photon } \\
\text { Detectors for } \\
\text { Enhanced } \\
\text { Exoplanet } \\
\text { Science }\end{array}$} & $\begin{array}{l}\text { Operational } \\
\text { Bandwidth }\end{array}$ & $200 \mathrm{~nm}-400 \mathrm{~nm}$ & \multirow{7}{*}{$\begin{array}{l}\text { GaN-based EBCMOS } \\
\text { and MCP detectors } \\
\text { meet required noise } \\
\text { specifications, but } \\
\text { require better } \\
\text { quantum efficiency to } \\
>50 \% \text { and } \\
\text { improvements in } \\
\text { lifetime } \\
\text { Superconducting } \\
\text { MKID and TES } \\
\text { detectors also apply } \\
\text { here }\end{array}$} & \multirow{7}{*}{$2-4$} & \multirow{7}{*}{$\begin{array}{l}\text { Technology, } \\
\text { Engineering, } \\
\text { Manufacturing }\end{array}$} \\
\hline & Read Noise & $\ll<1 \mathrm{e}^{-}$ & & & \\
\hline & Dark Current & $<0.001 \mathrm{e}^{-} / \mathrm{pix} / \mathrm{s}$ & & & \\
\hline & $\begin{array}{l}\text { Spurious Count } \\
\text { Rate }\end{array}$ & $\begin{array}{l}\text { Small compared to } \\
\text { dark current }\end{array}$ & & & \\
\hline & $\begin{array}{l}\text { Quantum } \\
\text { Efficiency }\end{array}$ & $\begin{array}{l}>50 \% \text { over the entire } \\
\text { band }\end{array}$ & & & \\
\hline & Format & $>2 \mathrm{k} \times 2 \mathrm{k}$ pixels & & & \\
\hline & Other & $\begin{array}{l}\text { Radiation hard, } \\
\text { minimum 5-year } \\
\text { lifetime at SEL2, } \\
\text { Non-cryogenic } \\
\text { operation preferable }\end{array}$ & & & \\
\hline \multirow{5}{*}{$\begin{array}{l}\text { Large-format } \\
\text { High- } \\
\text { Sensitivity UV } \\
\text { Detectors for } \\
\text { General } \\
\text { Astrophysics }\end{array}$} & $\begin{array}{l}\text { Operational } \\
\text { Bandwidth }\end{array}$ & $90 \mathrm{~nm}-300 \mathrm{~nm}$ & \multirow{5}{*}{$\begin{array}{l}\text { Same as above } \\
\delta \text {-doped EMCCD also } \\
\text { a candidate here, but } \\
\text { requires improved } \\
\text { radiation hardness } \\
\text { and reduction in } \\
\text { clock-induced charge }\end{array}$} & \multirow{5}{*}{4} & \multirow{5}{*}{$\begin{array}{l}\text { Engineering, } \\
\text { Manufacturing }\end{array}$} \\
\hline & Read Noise & $<5 \mathrm{e}^{-}$ & & & \\
\hline & $\begin{array}{l}\text { Quantum } \\
\text { Efficiency }\end{array}$ & $>70 \%$ & & & \\
\hline & Format & $>2 \mathrm{k} \times 2 \mathrm{k}$ pixels & & & \\
\hline & Other & $\begin{array}{l}\text { Radiation hard, } \\
\text { minimum 5-year } \\
\text { lifetime at SEL2, } \\
\text { Non-cryogenic } \\
\text { operation preferable, } \\
\text { Visible blind }\end{array}$ & & & \\
\hline
\end{tabular}




\section{Recommended Actions}

For all cases, we recommend close collaboration between government, academic, and industry partners to promote detector advancements. A near-term priority should be to radiation-test EMCCD technologies to determine suitability for ATLAST in a halo orbit at the Sun-Earth L2 point. Although WFIRST-AFTA is radiation testing EMCCDs now, the performance requirements for ATLAST are likely to be significantly more demanding. Additional development of HgCdTe APD, MKID, TES, and other technologies can be pursued via parallel paths, perhaps by several competing teams, with modest funding. Downselecting candidate technologies circa 2020 will help focus resources towards mission-specific milestones by 2024 .

In the event that superconducting detector technologies such as MKID and TES are selected, investment in ultra-lowvibration cooling technology will be critical to enabling the exoplanet science component of ATLAST.

\section{CONCLUSION}

We presented five key technology areas to enable a large UV-Optical-Infrared space telescope that is capable of a broad array of general astrophysics observations, as well as detecting and characterizing habitable exoplanets: Internal Coronagraphs, Starshades, Ultra-stable Large Aperture Telescopes, Mirror Coatings, and Detectors. A technology gap list is provided for each area, and the current TRL and gap type are provided for each technology component. We provided recommended actions for developing each technology.

Portions of this text were submitted in response to a call for white papers from the Cosmic Origins Program Analysis Group's Science Interest Group \#2: Ultraviolet-Visible Cosmic Origins Space-Based Science and Technology Development.

\section{REFERENCES}

[1] Committee for a Decadal Survey of Astronomy and Astrophysics; National Research Council, New Worlds, New Horizons in Astronomy and Astrophysics, The National Academies Press, Washington, D.C. (2010).

[2] C. Kouveliotou, J. Centrella, B. Peterson, et al., Enduring Quests, Daring Visions: NASA Astrophysics in the Next Three Decades, arXiv:1401.3741 (2014).

[3] J. Dalcanton, S. Seager, et al., From Cosmic Birth to Living Earths: The Future of UVOIR Space Astronomy, published by the Association of Universities for Research in Astronomy, www.hdstvision.org/report/ (2015).

[4] P. Hertz, "Planning for the 2020 Decadal Survey: An Astrophysics Division White Paper", January 4, 2015, available at science.nasa.gov/astrophysics/documents/.

[5] N. Rioux, L. D. Feinberg, D. C. Redding, and H. P. Stahl, "A future large-aperture UVOIR space observatory: reference designs," in UV/Optical/IR Space Telescopes and Instruments: Innovative Technologies and Concepts VII, Proc. SPIE, H. MacEwen and J. Breckinridge, eds., (2015), Vol. 9602, pp. 9602-8.

[6] C. C. Stark, A. Roberge, A. Mandell, and T. D. Robinson, "Maximizing the ExoEarth Candidate Yield from a Future Direct Imaging Mission," in Ap. J., 795, p. 122 (2014).

[7] M. N'Diaye, L. Pueyo, and R. Soummer, “Apodized Pupil Lyot Coronagraphs for Arbitrary Apertures IV: Reduced Inner Working Angle and Increased Robustness to Low-Order Aberrations," in Ap. J., 799, p. 225 (2015).

[8] R. G. Lyon, B. A. Hicks, M. Clampin, and P. Petrone III, "Phase-Occulting Nulling Coronagraph," submitted to Ap. $J$., preprint available at arXiv:1504.05747 (2015). 
[9] O. Guyon, P. Hinz, E. Cady, et al., "High Performance Lyot and PIAA Coronagraphy for Arbitrarily Shaped Telescope Apertures," in Ap. J., 780, p. 171 (2013).

[10] S. Seager, W. Cash, S. Domagal-Goldman, et al., "Exo-S: Starshade Probe-Class Exoplanet Direct Imaging Mission Concept Final Report," available at exep.jpl.nasa.gov/stdt/.

[11] NASA Robotic Refueling Mission, RRM Task Overview, ssco.gsfc.nasa.gov/rrm_tasks.html, accessed 24 July 2015.

[12] R. G. Lyon and M. C. Clampin, "Space telescope sensitivity and controls for exoplanet imaging," in Optical Engineering, 51, p. 011002 (2012).

[13] H. P. Stahl, L. D. Feinberg, S. C. Texter, "JWST Primary Mirror Material Selection," in Optical, Infrared, and Millimeter Space Telescopes, Proc. SPIE, J. C. Mather ed., Vol. 5487, p. 818 (2004). 